\title{
Correcciones en lengua: modos de intervención docente en los textos escritos por los alumnos
}

\author{
Stella Maris Tapia \\ Universidad Nacional de Río Negro, Argentina
}

Artículo recibido 5 julio 2015, aceptado 14 marzo 2016, versión final 10 octubre 2016 DOI: http://dx.doi.org/10.5565/rev/jtl3.640

\begin{abstract}
Resumen
Este artículo se enmarca en la investigación doctoral sobre las correcciones que los docentes realizan en los textos escritos por sus alumnos de español (primera lengua) en los últimos años de la escuela secundaria y el primer año de la universidad en Bariloche, Argentina. Desde la línea teórica del interaccionismo socio-discursivo nos preguntamos cómo corrigen los docentes de las asignaturas Lengua y Literatura y afines. El análisis de los modos de corrección se basa en diferenciar tres maneras de intervención docente: enunciados, marcas y enmiendas, como intervenciones didácticas superpuestas en los textos de los alumnos. En la primera parte, se caracteriza cada una de estas categorías. En la segunda parte, se describe, a partir del concepto de "palabra ajena" de Voloshinov (1929), cómo las voces de los docentes interactúan con las voces de los alumnos.
\end{abstract}

Palabras clave: Lengua y Literatura, corrección, enunciado, marca, enmienda

\begin{abstract}
This article is part of the doctoral research on teachers' corrections of texts written by their students of Spanish L1 in the final years of high school and the first year of college in Bariloche, Argentina. Within the socio-discursive interactionism framework, we inquire how teachers correct written texts in subjects such as language, literature and related studies. The analysis of correction methods is based on differentiating three teachers' intervention approaches: statements, marks and amendments, as educational interventions superimposed over students' texts. In the first part, each of these categories is characterized. In the second part, it is described how teacher and student voices interact following Voloshinov's concept of the "foreign word" (1929).
\end{abstract}

Keywords: Language and literature, correction, statement, mark, amendment

\section{Résumé}

Cet article fait partie d'une recherche de doctorat sur les corrections que les enseignants font des textes écrits par leurs apprenants d'espagnol (langue première) au lycée et dans la première année de l'université à Bariloche en Argentine.

A partir de l'approche théorique de l'interactionnisme socio-discussif on se demande comment corrigent les enseignants des matières de Langue et Littérature et autres liées au même sujet. L'analyse des méthodes de correction est basée sur la différenciation de trois formes d'intervention de l'enseignant: énoncés, marques et rectifications, en tant qu'interventions didactiques superposées dans les textes des apprenants. Dans la première partie on caractérise chacune des catégories. Dans la seconde partie on décrit, à partir du 
concept de « mot étranger» de Voloshinov (1929), la manière dont les voix des enseignants interagissent avec les voix des apprenants.

Mots clés: Langue et littérature, correction, énoncé, marque, rectification.

\section{Introducción}

La investigación realizada en el marco de la tesis doctoral "La corrección de textos escritos: qué, cómo y para qué se corrige en Lengua" (Universidad Nacional de Córdoba, Argentina, defendida en diciembre de 2014) tuvo por objetivo conocer y describir qué corrigen los docentes en las asignaturas en las clases de lengua (español L1) y materias afines, en los últimos años de la escuela secundaria y en el inicio de los estudios universitarios en San Carlos de Bariloche, Argentina, para caracterizar el modo en que tales correcciones se llevan a cabo.

El presente artículo sintetiza un aspecto parcial de dicha investigación: se propone describir los modos en los que los docentes corrigen los textos escritos por sus alumnos desde el análisis textual para señalar la presencia o ausencia de indicadores de los efectos que los docentes esperan y desean conseguir sobre el desarrollo de las capacidades discursivotextuales de sus alumnos.

Consideramos a las correcciones como acciones comunicativas de intervención didáctica en la enseñanza de la lengua. La ambigüedad del término "corregir", asociado en el sentido común con el error y con un señalamiento de la forma correcta, requiere una primera aclaración: la distinción entre el uso cotidiano y el uso del término en ámbitos educativos. Interesa relevar, para esta investigación, lo que los profesores identifican como corrección: una práctica en y sobre los textos de sus alumnos. Para ello se define la unidad de análisis "corrección" como un enunciado, una marca verbal, una marca gráfica o la combinación de dos o más de ellos realizados por un docente sobre el enunciado (producto de la actividad de lenguaje) de cada uno de los alumnos guiado por una consigna.

La corrección se relaciona con un proceso de evaluación en un sentido amplio, en tanto que supone un juicio de valor sobre el producto de la acción del lenguaje (texto) de un alumno en su carácter de autor. El docente identifica zonas de los textos de los alumnos que requieren de su intervención, y en este sentido la corrección se asocia con la formulación de juicios y valoraciones (aunque no necesariamente con juicios negativos) y, por lo tanto, con la evaluación; sin embargo, el deslindar evaluación de corrección permite centrarnos en esta última como objeto de estudio, en tanto acción que los docentes realizan, a manera de diálogo didáctico, sobre los textos escritos previamente por sus alumnos. En la investigación se 
procura conocer las dimensiones de la corrección en su aspecto comunicativo, sin confundirlas con las dimensiones evaluativas que dan cuenta de cómo ha valorado un docente el producto de la acción de lenguaje del alumno.

\section{Marco teórico: los textos como producto de la actividad del lenguaje y las intervenciones didácticas como mediaciones formativas}

El marco teórico en el que nos inscribimos es el interaccionismo socio-discursivo, que sintetiza los aportes teóricos de Voloshinov y Vigotski ${ }^{1}$, autores referenciales para definir al lenguaje como actividad humana y a la interacción dialógica como principio de esa actividad. Los textos, productos empíricos de la actividad de lenguaje (Bronckart, 2004), son construidos en el marco de interacciones semióticas en una generación constante. Voloshinov (1929/2009) considera que el lenguaje es creación libre y activa, puesto que constantemente se generan enunciados. Cada enunciado es una unidad producida en la interacción dialógica e ideológica del individuo con su medio, como el eslabón de una "cadena ininterrumpida" (Voloshinov, 1929/2009, p. 116). Esta cadena no puede ser comprendida y explicada sino en relación con un contexto, una situación concreta de comunicación, ya que los enunciados se orientan hacia las actuaciones de la comunicación verbal, anteriores en el tiempo, históricas, de donde toman sus condiciones genéricas.

En este sentido, el diálogo, para Voloshinov, es la forma característica de interacción por la palabra, por lo que los enunciados se organizan en función del auditorio y de su respuesta, esto es, la evaluación o valoración compartida con el hablante. De allí la relevancia de la palabra ajena, por la que está conformado cada enunciado y a la que cada uno se orienta.

El lenguaje, en el proceso histórico de la socialización es concebido, siguiendo a Vigotsky (1930 y 1931/2008), como el origen de las propiedades de la conducta y de la conciencia humana, en tanto el individuo se apropia e internaliza los signos y los significados que reorganizan sus funciones psíquicas propiciando el desarrollo. El desarrollo humano se explica mediante el signo, medio para realizar una acción psíquica que posibilita el desarrollo de operaciones. Entre los signos, es, para Vigotski (1934), la palabra la que, a través del significado, da realidad al pensamiento, reestructurando la conciencia en un proceso dinámico. Y esta palabra es la que proviene del medio exterior, de la interacción con otros en el lenguaje. El medio social y la actividad del lenguaje constituyen, tanto para Vigotski como para Voloshinov, la génesis de la conciencia humana.

La articulación que realiza el interaccionismo socio-discursivo (Bronckart, 2004, 2007) de la teoría de Voloshinov y Vigotski, entre otros autores, se propone como una síntesis 
orientada a las mediaciones formativas y como un referente constituyente del campo de la didáctica de la lengua y la literatura. Su programa de trabajo descendente parte del nivel de los preconstructos históricos humanos y sus modalidades particulares para abordar cómo se transmiten y reproducen esos preconstructos y cómo se enfocan los efectos de esa transmisión en la constitución de las personas.

Las mediaciones formativas se consideran, en esta teoría, procesos conscientes en los que los adultos integran a las nuevas generaciones en actividades conjuntas, transmitiendo y explicándoles normas, valores sociales y conocimientos. Interesan particularmente los procesos de educación formal y sus dimensiones didácticas y pedagógicas, centrados en la enseñanza y el aprendizaje de la textualidad o, en otras palabras, en una "didáctica del texto" (Bronckart, 2007).

Como espacios de interacción didáctica, consignas de enseñanza y correcciones constituyen mediaciones formativas materializadas en productos textuales: mientras que las consignas son acciones de lenguaje con la finalidad específica de guiar las diversas acciones de lenguaje de los alumnos en el proceso cultural de interiorización de las capacidades lingüísticas (Riestra, 2004), las correcciones que los docentes realizan son instrumentos con los que se analiza y valora la acción verbal desplegada. Reconocer las actividades externas observables en situaciones didácticas (Dolz y Erard, 2000) es condición necesaria y previa a cualquier intento de proponer cómo las mediaciones formativas pueden internalizarse y afectar al desarrollo de las capacidades discursivas y textuales de los alumnos. En este sentido se procura deslindar mediante el análisis de textos corregidos qué efecto formativo supondrían ciertos modos de corregir para el desarrollo de capacidades discursivo-textuales.

\section{Metodología de recolección y análisis del corpus}

La metodología de la investigación es situada e interpretativa. El corpus de trabajos corregidos por distintos profesores de nivel medio y de cursos preuniversitarios se constituyó mediante el pedido explícito a los diferentes docentes, con las indicaciones de que fueran trabajos de escritura de los alumnos que respondieran en cada grupo a una misma consigna de trabajo y que la muestra reflejara la situación media del curso. Se recolectaron 128 textos corregidos por doce docentes que se desempeñaron en la materia Lengua y Literatura en los últimos tres años de la escuela secundaria, en instituciones públicas y privadas ${ }^{2}$ de $\operatorname{San}$ Carlos de Bariloche, y 122 corregidos por doce docentes que dictaron Introducción a la Lectura y Escritura Académica (ILEA), de la Sede Andina, Universidad Nacional de Río Negro, y Usos

y formas de la Lengua Escrita, del Centro Regional Universitario Bariloche, de la 
Universidad Nacional del Comahue. Estas últimas son materias del inicio de los estudios superiores. La muestra así conformada busca representar ecológicamente un estado de enseñanza de la lengua que comprende el $40 \%$ de las instituciones secundarias y la totalidad de las universidades públicas de la región que cuentan con materias del área en su primer año de formación. La selección de los dos niveles obedece a un interés teórico por estudiar las prácticas docentes en la transición entre la educación obligatoria y el nivel universitario, aspecto que no se abordará en el presente artículo.

Los tipos de análisis que se realizan son el análisis textual de las correcciones y el análisis a partir de la consigna. En el primer tipo, siguiendo la arquitectura textual (Bronckart, 2004) se busca poner en evidencia: infraestructura general (secuencias, por ejemplo instruccionales), mecanismos de textualización, mecanismos de asunción de la responsabilidad enunciativa (modalizaciones y voces empleadas) en los enunciados de las correcciones. El análisis a partir de la consigna (Riestra, 2004) articula la actividad, la tarea realizada con esa consigna y las correcciones que dan cuenta tanto de la evaluación de la tarea como de los contenidos de la enseñanza de la lengua y de nuevas "instrucciones" en el uso de la lengua.

\section{Los modos de inserción de las correcciones en los textos de los alumnos}

Se recolectaron conjuntos de 10 a 20 textos que responden a una misma consigna de trabajo, corregidos por cada docente. El análisis de las consignas, de los objetos de enseñanza y de los objetos de corrección nos permitió registrar qué se corrige como primer nivel de análisis, distinguiendo correcciones en el nivel global de los textos (consigna, género textual, aplicaciones de los contenidos desarrollados durante las clases, entre otras), en su sentido (congruencia con los mundos representados, planificación general y progresión de la información, coherencia temática marcada por mecanismos de textualización) y en su forma (puntuación, morfosintaxis, léxico, ortografía). Los diferentes aspectos corregidos son independientes de las consignas de trabajo y de las distintas tareas que las consignas prescriben, porque los docentes corrigen interviniendo en todos los niveles de los textos escritos por los alumnos.

Pudieron distinguirse diferentes modos de intervención docente o modos de corregir que delimitaremos a partir de la combinación de tres categorías: enunciados, marcas y enmiendas. En esta primera parte diferenciamos cada una de las formas, presentando ejemplos extraídos del corpus. Para que esta ilustración muestre el entramado de las voces de docentes $\mathrm{y}$ alumnos, se seleccionaron fragmentos que fueron reproducidos digitalmente. Entre 
paréntesis se colocan las siglas de la materia escolar o asignatura universitaria a la que corresponde esa producción textual. El número antepuesto a la sigla identifica al docente que corrigió.

\section{Las correcciones mediante enunciados}

Los enunciados, específicamente, adoptan las formas de réplicas destinadas a los alumnosproductores de cada texto. Dan cuenta de la relación social inmediata entre los participantes de la acción comunicativa, el docente y el alumno, en una situación social más amplia y englobadora como son la institución escolar o la institución académica. El contexto de estos enunciados está determinado, asimismo, por las tareas dirigidas por una consigna de trabajo (Riestra, 2004). Consisten en valoraciones o juicios, instrucciones y preguntas retóricas. Su contenido temático concierne a los objetos de enseñanza y las acciones y las operaciones que los alumnos han internalizado o que sería deseable que hubieran internalizado en las clases de Lengua. Construyen tanto una configuración del destinatario como del enunciador y revelan, en primer plano, las representaciones que el docente hace de sí mismo en su rol de profesor y de los alumnos como destinatarios.

Los ejemplos 1 y 2 ilustran el entramado de enunciados y marcas de corrección. En el 1, pueden observarse tres enunciados de la docente. El primero de ellos se refiere a un aspecto global que podemos considerar estilístico: la preferencia por el verbo "decir", en su forma sintética, conjugado en futuro simple del modo indicativo en lugar de la forma analítica y perifrástica usada por el alumno- propia del español americano. Otro enunciado global se refiere al género textual y el tercero, al sentido del texto, de modo general: "ajustar coherencia en 2". Asimismo, la docente ha subrayado las expresiones "voy a decir que" y "en el cual": mientras que la primera de estas dos marcas se corresponde con el primer enunciado, no hay un enunciado que informe acerca de lo señalado en el segundo caso. Además, sustituye la forma "voy a decir que" por "diré que".

Ejemplo 1. Corrección mediante enunciados

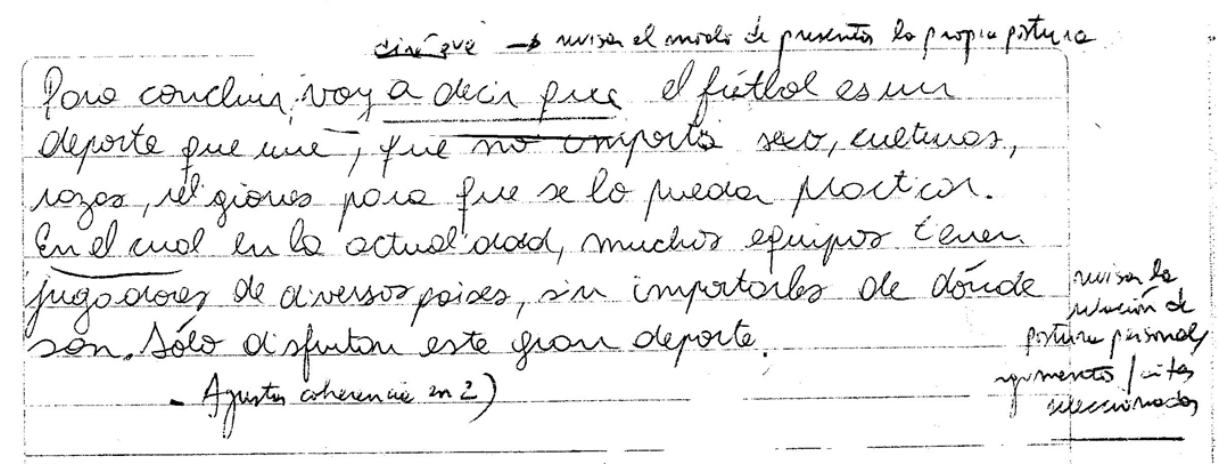


Transcripción: "revisar el modo de presentar la propia postura", "revisar la relación de postura personal, argumentos, citas seleccionadas", "Ajustar coherencia en 2)" (3 ILEA 1).

En el ejemplo 2, pueden observarse dos enunciados del docente; el primero de ellos indica un aspecto procedimental (“justificar con la frase"), que se relaciona directamente con la consigna de trabajo, en donde se solicita al alumno la transcripción una cita para fundamentar una afirmación. Con el segundo enunciado se identifica un problema de sentido. Mientras que el primer enunciado emplea una forma en infinitivo con valor de imperativo, el segundo de ellos es predicativo, se infiere que lo que "no es claro" es el enunciado escrito por la alumna, puesto que no hay, en él, ningún antecedente con el que concuerde el adjetivo "claro".

Ejemplo 2. Corrección mediante enunciados.

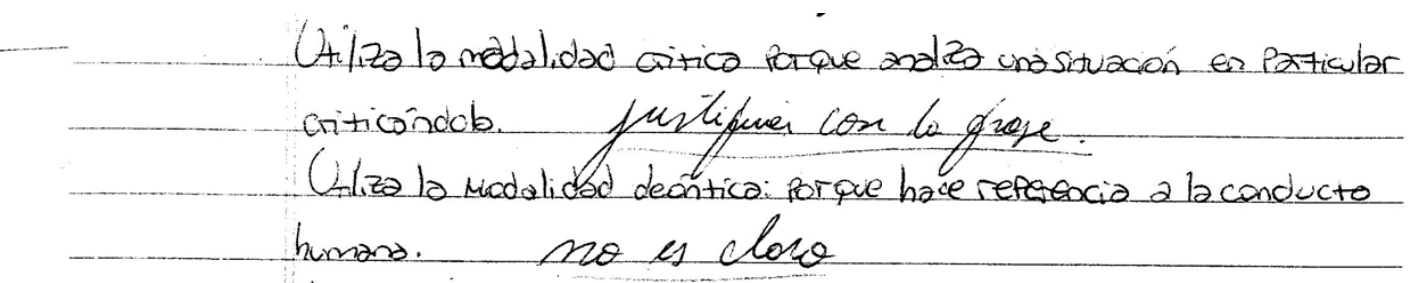

\section{Transcripción: "justificar con la frase", "no es claro" (2 NS 2).}

Atendiendo a la ausencia o la presencia de marcadores (pronombres personales de segunda y tercera persona, formas verbales) que reflejan la interacción entre docente y alumnos, y el empleo de los mecanismos de asunción de la responsabilidad enunciativa (Bronckart, 2004), en los enunciados se pueden distinguir formas más objetivadas o distantes y formas subjetivadas o de acercamiento con los alumnos.

Las formas afectivas o subjetivadas son aquellas en las que el autor de la corrección involucra a su destinatario, por ejemplo, mediante el empleo de pronombres personales y vocativos, el uso del nombre propio y/o de apodos del alumno para dirigirse a él, y el empleo de verbos en segunda persona del singular o en primera persona del plural que incluye al docente (Por ejemplo: “Ana, revisá 3 esto", "lo charlamos"). Mediante estas formas afectivas se buscaría una proximidad con el alumno, personalizando la corrección y apelando a las emociones compartidas que se ponen de relieve en la interacción. La ausencia de estos marcadores y el empleo de mecanismos con valor de impersonalidad (como los imperativos, en los ejemplos precedentes) en cambio, tenderían a producir un efecto de distanciamiento entre el docente y el alumno-destinatario de la corrección, porque el contenido de las correcciones se presenta objetivado o autónomo de la interacción docente-alumno. Este 
distanciamiento podría resultar de la pretensión de mostrar la corrección como parte de una evaluación imparcial y despojada de toda implicación emocional. Ambas formas se relacionan con los estilos personales de cada docente antes que con los contenidos de enseñanza y con las consignas de trabajo que motivan las correcciones.

\section{Las marcas como subcódigos de corrección}

En relación con las marcas que los docentes realizan como correcciones de las tareas de los alumnos, señalamos la presencia de signos que pertenecen a subcódigos no articulados y no uniformes, que cada docente emplea de manera personal, según sus propios criterios y estilos.

Las marcas conforman sistemas de representación secundarios de código no lingüístico que interviene en el código lingüístico. Combinadas con signos lingüísticos que las explican, forman parte de códigos complejos. Entre las más usadas, diferentes modos de subrayado, corchetes y redondeos adoptan distintos significados para cada uno de los docentes inclusive con significados variados en la corrección de un único texto; en líneas generales, identifican errores.

El signo de interrogación es empleado con distintos fines, entre los que se destaca su uso para dar cuenta de la incongruencia entre lo enunciado por un alumno y el mundo representado, omisiones de información o errores en su planificación (por ejemplo, por reiteraciones de palabras), y caligrafía defectuosa, que dificulta la legibilidad.

Del análisis del corpus se observa que la mayor complejidad del sistema de corrección mediante marcas (códigos conformados por mayor cantidad de signos) no reduce el grado de sinonimia, sino que para un mismo tipo de error son usadas dos, tres o más marcas como sinónimos, así, se emplean diferentes formas de subrayados para el mismo tipo de error, por ejemplo, para errores ortográficos. En el ejemplo 3 un círculo vacío a la altura de la línea del renglón indica la omisión de un signo de puntuación; una cruz debajo de una vocal refuerza el error enmendado por la docente, consistente en la omisión de una tilde; el subrayado simple bajo el pronombre "la cual" se combina con el subrayado de "ilustrada" y con una línea sinusoidal al margen para dar cuenta de errores de sentido. Una cruz dentro de un círculo se agrega a un pronombre redondeado y se explicita "sintaxis". Una flecha hacia arriba podría interpretarse como la necesidad de un reordenamiento en la organización de la información y se superpone con la línea sinusoidal que señala la coherencia. Asimismo, la línea sinusoidal subrayando una palabra se complementa con las palabras "tiempo verbal" para indicar en qué consiste el error de coherencia. Los significados de estas marcas se superponen parcialmente. 
Ejemplo 3. Corrección mediante marcas no articuladas combinadas con signos articulados (lingüísticos); significados superpuestos (1 ILEA 2).

$$
\text { unveries y ivorinuraries. }
$$

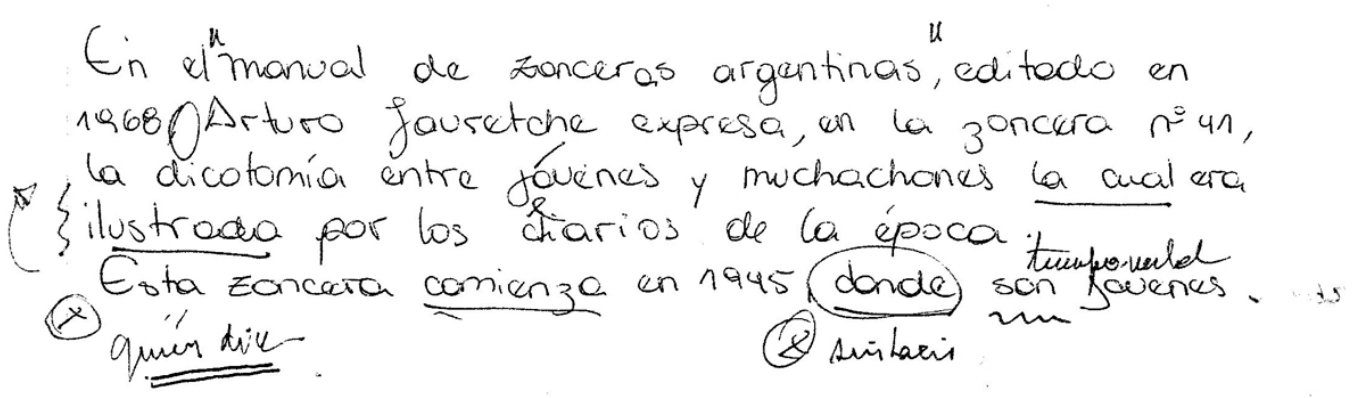

¿Cuál es el objetivo de emplear marcas durante la corrección? ¿Qué efecto buscarían los docentes al usarlas? Si el objetivo es que los alumnos reconozcan el significado al leer los textos corregidos, el sistema requiere una serie de restricciones: en primer lugar, un código de marcas finito (admitiendo un número finito de signos, como de hecho vemos en los textos corregidos por los docentes, que contemplan un máximo de ocho signos). En segundo lugar, los significados de esos signos, una vez dados, no podrían variar de un contexto a otro (de un texto corregido a otro). En tercer lugar, la relación entre significante (tipo de marca, sea ésta subrayado, círculo, signo de interrogación, etc.) y significado (verbigracia, error cometido en un nivel del texto del alumno) debería ser unívoca. Por último, debiera tender a evitar la sinonimia, es decir, reducir la creatividad del código.

Cuando las marcas son empleadas sistemáticamente, y lo marcado de una manera tiene un significado distinto de lo marcado de otra forma, orientarían a su destinatario acerca del nivel específico de los textos en los que el docente considera necesario intervenir. Cuando las marcas no conforman un código, es decir, no hay una relación unívoca entre marca utilizada y significado en el nivel del texto en el que el docente quiso intervenir, no podría concluirse que el efecto pretendido por éste sea que el alumno busque el sentido, sino que la marca funcionaría como un paratexto o medio complementario de la lectura que pretende llamar la atención, y ello explicaría su frecuente redundancia con enunciados y/o enmiendas como modo de corregir. Éste tipo de uso de las marcas es el predominante en el corpus, donde se observa, además, que cada uno de los docentes emplea códigos personales, sin sistematicidad ni estabilidad de un texto a otro o en el mismo texto.

\section{Las enmiendas}

La enmienda como categoría da cuenta de la intervención directa del docente sobre el enunciado ajeno que se encuentra o percibe como erróneo. La palabra "enmienda" deriva, 
etimológicamente, de eméndare, "corregir las faltas", que proviene, a la vez, de menda y mendum, "falta, error, defecto" (Coromines, 2009). La definición del diccionario de enmendar es "corregir, sacar defectos" (RAE, 2001). La enmienda implica la reposición, a cargo del docente, de los signos lingüísticos y signos de puntuación, de las tildes y de los grafemas omitidos, el tachado de signos o elementos de más y la sustitución de un elemento por otro.

Las enmiendas logran una versión nueva del texto a dos voces o co-rregido (en el sentido etimológico de corregir, "reinar con"- Coromines, 2009-, es decir, con dos rectores o dos autores cogobernando el texto).

En el ejemplo 4 puede observarse cómo la profesora enmienda el texto (además de marcar los errores mediante subrayados y cruces): coloca el signo de puntuación omitido (coma); repone el verbo (era), traza las tildes y tacha una letra h usada en el verbo "ir".

Ejemplo 4. Enmiendas en aspectos de forma (puntuación, sintaxis, ortografía) (9 NS 4).

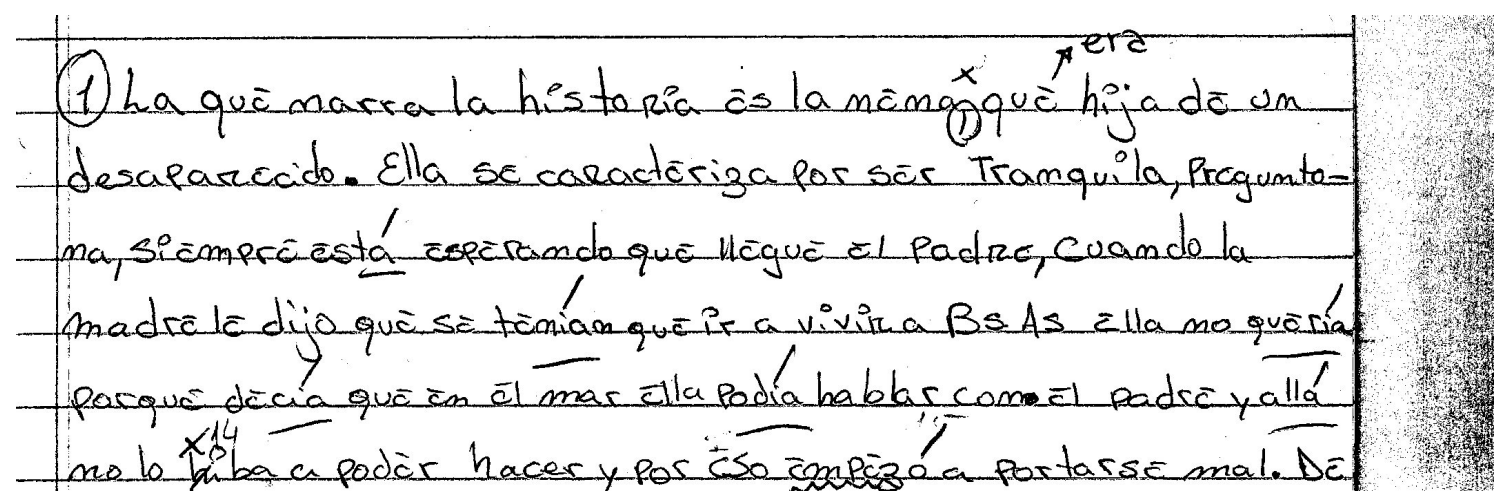

En el ejemplo 5, la docente, además de reponer una tilde, agregar un pronombre relativo, ubicar en su forma plural un verbo para mantener la concordancia, colocar un punto omitido y tachar una palabra, agrega información y un organizador textual para marcar una relación de sentido a nivel inter-oracional. Se ven aquí enmiendas tanto en el nivel de la forma (puntuación, morfología, sintaxis, ortografía) como en el nivel del sentido.

Ejemplo 5. Enmienda referida al sentido: progresión de la información (interoracional).

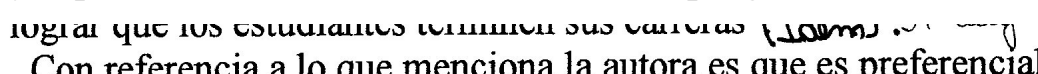

Con referencia a lo que menciona la autora es que es preferencial mantener la matrículaty,yơ se tomanen cuenta en América Latina las posiciones de los ranking. Se trata más bien de imponer modos de estudio para que los ingresantes a las universidades entren con un nivel nivataxio básico para continuar con la carrera y no abandonarla, Se enfocan en ayudar más a los estudiantes desde ese punto de vista.

En el artículo se señala que "el mundo desarrollado es el mayor

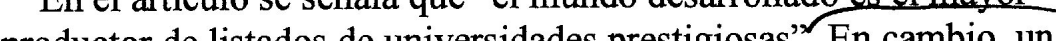


Transcripción: "En este sentido, las univ. locales se enfocan en ayudar más a los estudiantes desde ese punto de vista" (9 ILEA 7).

¿Cuál es el efecto que podría tener el uso extendido de la enmienda como modo de corregir? ¿Cómo se puede explicar la presencia simultánea y redundante de marcas y de sustituciones, reposiciones y tachaduras? El efecto que buscaría provocar la redundancia de marcas y enmiendas podría asimilarse a lo que, en la teoría de la actividad de Leontiev, se denomina presentación y que consiste en la toma de conciencia o transformación del reflejo psíquico inconsciente en reflejo consciente. Según Leontiev (1983), las acciones se transforman en operaciones cuando diferentes acciones parciales se fusionan en una acción única. En tal caso, la acción y su objetivo no se "presentan" directamente en la conciencia: el contenido consciente se vuelve contenido inconsciente.

Las operaciones conscientes se forman primero como unos procesos apuntando a un objetivo, que sólo después, en algunos casos, pueden adquirir la forma de prácticas automatizadas. (Leontiev, 1983, p. 246)

Una acción se transforma en operación (habilidad y costumbre) si pasa a ser condición de una nueva acción requerida por un nuevo objetivo. La operación constituye un paso inferior en la estructura de la actividad, un requisito para realizar una acción, sin implicar que esa operación se simplifique:

Al convertirse en operación, sale de la esfera de los procesos concienciados, pero conserva las características fundamentales del proceso consciente y puede, en todo momento, en caso de dificultad, por ejemplo, ser de nuevo concienciada. (Leontiev, 1983 , p. 252)

Por medio de la "presentación", la operación puede volverse acción consciente, al develar los sentidos de un fenómeno mediante la designación, es decir, encarnándolos en significaciones que concretizan los sentidos.

Considerando ahora las enmiendas en los textos de los alumnos, estas podrían tener el efecto de presentación en tanto su finalidad es la toma de conciencia, por parte del agente productor del texto escrito corregido, del sentido personal de las acciones que conforman la actividad de escritura y de las operaciones, en ella implicadas, de combinación y selección de los recursos de la propia lengua. En particular, la redundancia de marcas y enmiendas tendría el efecto de indicar el error y su solución, volviendo conscientes operaciones ya internalizadas por los alumnos en relación con la escritura.

Ninguno de los textos corregidos presenta la enmienda como la única forma de corrección, antes bien, las tres categorías que se han descripto aparecen combinadas, no hay correspondencias unívocas entre niveles de corrección de los textos y formas de corregir e 
incluso se observan situaciones en las que un profesor, en el mismo texto de un alumno y ante un tipo de error similar, lo subsana una vez con una enmienda y luego marcando o bien, identificándolo mediante un enunciado, como puede verse en el ejemplo 6. Allí, el uso de marcas -una flecha acompañada por el enunciado "concordancia" y un subrayado para una preposición usada incorrectamente ("pertenecen en" en lugar de "pertenecen a")- como correcciones en el nivel sintáctico, se conjuga con la enmienda de otros errores sintácticos mediante la reposición de verbo, artículo y pronombre.

Ejemplo 6. Superposición de modos de corregir en una misma tarea y para el mismo tipo de errores (5 ILEA 3).

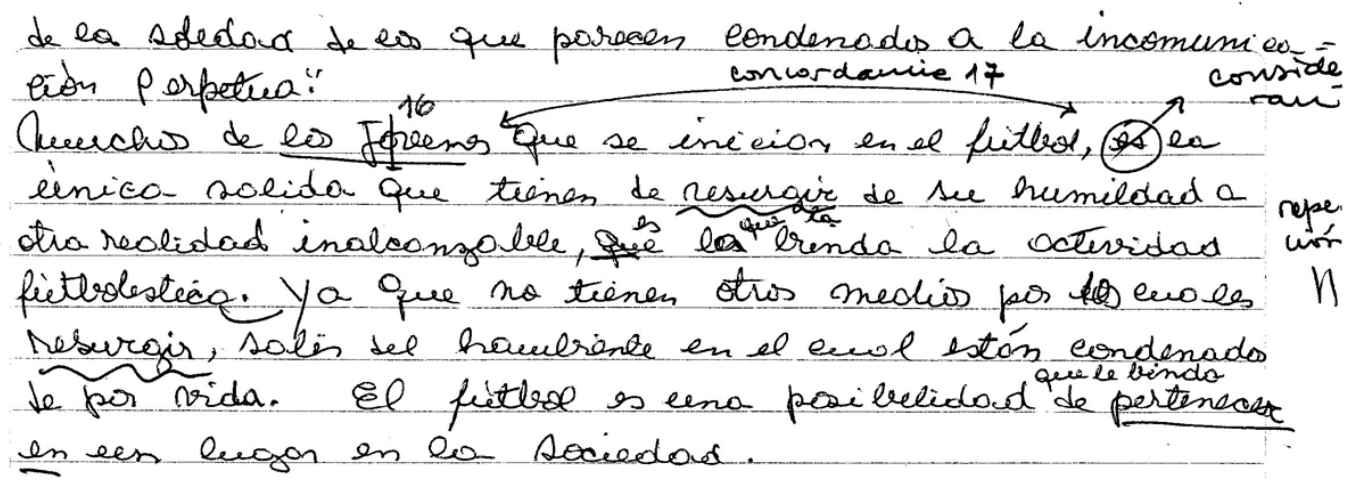

En este sentido, enunciados, marcas y enmiendas no se tratan de categorías mutuamente excluyentes, sino, antes bien, funcionan como descriptores para analizar los modos de corregir, ya que se combinan en una misma indicación del docente o ante un mismo error del alumno, generando un efecto de redundancia, como se ilustra en el ejemplo 7.

Ejemplo 7. Simultaneidad de modos de corregir en una misma tarea: redundancia de marcas (subrayado) y enmiendas para errores de ortografía y redundancia de marcas (círculo, subrayado) y enunciados para sintaxis y sentido.

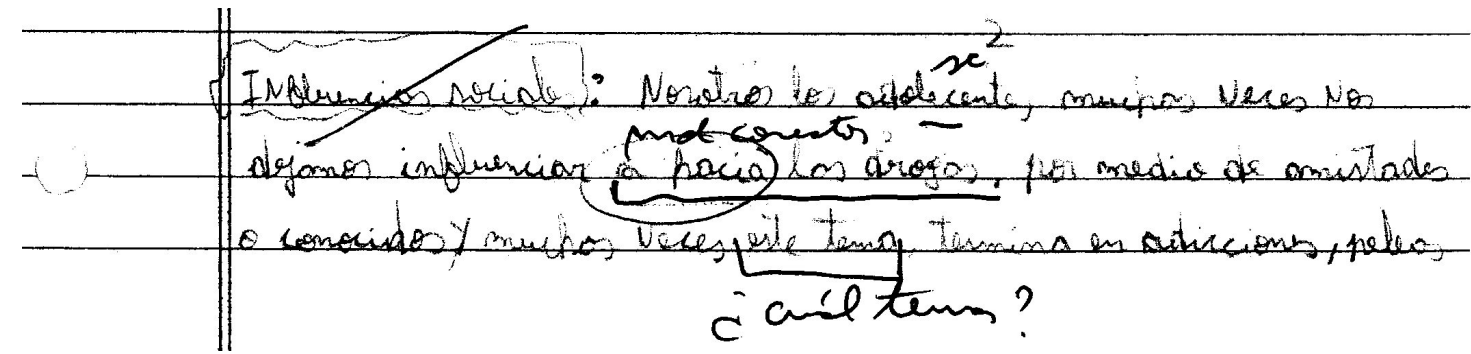

Transcripción: “sc”, “mal conector” y “¿cuál tema?” (9 NS 13).

Por tratarse de categorías de análisis superpuestas y simultáneas, estos datos no son cuantificables. Por otra parte, la simultaneidad nos obliga a preguntarnos: ¿qué efecto busca el 
docente mediante la reiteración de indicaciones realizadas de diferentes maneras? ¿Cuál es el objetivo de reforzar las correcciones mediante dos o tres modos distintos? ¿Existe una finalidad o es simplemente una acción realizada sobre el objeto empírico-texto como espacio específico de la tarea docente?

\section{El diálogo docente- alumno en la corrección: los modos de intervención}

Para completar la caracterización de los modos de corregir anteriores, proponemos retornar a las definiciones de diálogo y de palabra ajena de Voloshinov (1929), puesto que permiten explicar la interrelación y la inserción de enunciados de diferente autoría en un texto. La caracterización de los modos de corrección a partir de la teoría de Voloshinov sobre la interacción en el lenguaje -erróneamente atribuida a Bajtín (Bota y Bronckart, 2010)- fue señalada por Ruiz (1998), quien abreva de las categorías bajtinianas de polifonía y de monologismo, para referirse a los estilos de profesores corrigiendo. Aunque es Ruiz un antecedente de la presente investigación por trabajar con un corpus de textos corregidos enfocando el problema de la corrección para el portugués lengua primera, el análisis de los datos de profesores brasileros corrigiendo da cuenta de una mayor homogeneidad que la que identificamos en Bariloche. La autora revela, además, una correspondencia entre qué se corrige y cómo se hace, que no hemos registrado en nuestro corpus, antes bien, como ya se mencionó, no hay en los trabajos corregidos una relación directa entre qué se corrige y el modo de corregir. Por otra parte, dada la autoría de Voloshinov de los conceptos teóricos, consideramos necesario revisar la lectura de El marxismo y la filosofía del lenguaje no mediada por los textos de Bajtín y los conceptos de polifonía e intertextualidad con ellos relacionados.

Voloshinov advierte que todos los enunciados, productos de la generación continua en el lenguaje, están orientados hacia sus destinatarios específicos, ya que la palabra, en la interacción entre quien la enuncia y su interlocutor, se determina por ambas partes. Cualquier interacción comunicativa está orientada hacia su destinatario: cualquier acción en el lenguaje es una interacción que prevé su respuesta y que se inserta en la comunicación ininterrumpida. No obstante, Voloshinov señala un carácter peculiar de ciertas interacciones que, además de tener este carácter dialógico general, se distinguen por el uso de la palabra del otro -o palabra ajena- en el propio enunciado.

La "palabra ajena” es “...un discurso en el discurso enunciado dentro de otro enunciado, pero al mismo tiempo es un discurso enunciado acerca de otro enunciado" (Voloshinov, 1929, p. 180), es decir, es un enunciado referido dentro de un enunciado autoral. 
El autor agrega: "El discurso ajeno y el contexto transmisor no son más que los términos de una interrelación dinámica. Esta, a su vez, refleja el dinamismo de la orientación social recíproca de las personas en el proceso de la comunicación ideológico-verbal" (Voloshinov, 1929, p. 186).

Las correcciones de los docentes se insertan en los enunciados de los alumnos, es decir, se realizan en un enunciado ajeno, con el que conforman un tipo especial de diálogo o interrelación. Se conforman con y sobre la palabra ajena: con los enunciados de los alumnos y sobre ellos.

Voloshinov distingue dos modos de inserción de la palabra ajena en un contexto autoral: el estilo lineal o dogmático y el estilo pictórico. El estilo lineal traza los límites entre el enunciado ajeno y el enunciado autoral: "Su tendencia principal consiste en la creación de contornos externos nítidos en discurso ajeno débil en su individuación" (Voloshinov, 1929, p. 188). El estilo pictórico, en cambio, no delimita la separación entre palabra ajena y contexto autoral:

Su tendencia consiste en desdibujar los nítidos contornos exteriores de la palabra ajena. En este caso el mismo discurso aparece individualizado en una medida mucho mayor. (Voloshinov, 1929, p. 189)

Esta última forma borra las fronteras del discurso ajeno.

Para realizar una analogía entre la incorporación del discurso ajeno y las correcciones se requiere invertir las relaciones que marca Voloshinov en el tratamiento de las fronteras entre ambos enunciados, puesto que la inserción de una voz segunda o voz del docente se realiza sobre la voz primera o voz del alumno, no refiriendo ese texto previo, sino replicándolo. Algunas de las características que Voloshinov especifica como la reacción de una palabra a la otra en la introducción de palabras ajenas en un enunciado explican los rasgos de las correcciones: la no disociación, gramaticalmente, de enunciados de profesores y alumnos; la incorporación de ambos en un contexto unificado y la ausencia de las formas sintácticas que estructuran la unidad del diálogo. La fusión gramatical entre dos enunciados autorales podría ser la causa de que gran parte de las correcciones de los docentes no comiencen con mayúscula, lo que refuerza su carácter dependiente con respecto a los enunciados de los alumnos.

Enunciados y marcas constituyen modos del estilo pictórico, caracterizado por sus relativismos y matices evaluativos. Son formas en las que los docentes mantienen una distancia y una diferenciación autoral con los enunciados de los alumnos: señalan las 
características del discurso ajeno e identifican sus errores y problemas pero ambas voces, la del docente y la del alumno, conservan sus fronteras diferenciadas.

El estilo lineal o dogmático de las correcciones -retomando aquí la caracterización de la inserción de la palabra ajena de Voloshinov- supone un docente que se apodera del enunciado del alumno para sustituirlo y alcanzar una versión nueva, "sin defectos", según la definición de enmienda de la RAE (2001). La enmienda indica que ambas voces se entremezclan y fusionan. Se borran las fronteras entre el discurso ajeno y el contexto autoral y el producto de la corrección es un único texto enmendado. El uso de las enmiendas como modo de corregir aumenta el efecto de impersonalidad de la corrección y contribuye a su objetivación, en tanto que la voz del docente borra sus límites con la voz del alumno; ambas voces se integran en un texto con dos contextos de producción. Mientras que la enmienda podría tener un efecto de presentación en el nivel de la forma, en el plano del sentido el borramiento de los límites de las voces autorales generaría el efecto de clausura del diálogo sobre el texto escrito, porque una de las voces autorales, la del docente, se apoderaría del sentido del texto del alumno. En este último caso, el efecto de la enmienda sería contrario a promover una reescritura del texto.

\section{Conclusiones}

Enunciados, marcas y enmiendas son tres modos que los docentes combinan para corregir los textos de sus alumnos, sin que exista una relación directa y unívoca, aplicada sistemáticamente, entre qué y cómo se corrige.

Así como los textos empíricos presentan niveles entramados e imbricados, las correcciones que los docentes realizan sobre ellos buscan dar cuenta de esa complejidad atendiendo numerosos aspectos a la vez, sin que estén sistematizados los modos de corregir en función de los objetos de corrección. Se ha notado cierta redundancia consistente en la combinación y simultaneidad de distintos modos de corregir para señalar un mismo error. Esta redundancia implica que marcas y enunciados operan como refuerzos de enmiendas y viceversa y produce un efecto visual de refuerzo que revela la preocupación de los docentes por verbalizar, en el diálogo establecido por las correcciones, aquello que consideran significativo para los aprendizajes de los alumnos.

En las interacciones entre docente y alumnos, las réplicas que los docentes realizan en el diálogo escrito en los textos procuran encontrar modos de generar un efecto en sus destinatarios. El análisis textual realizado, no obstante, permite dar cuenta de una serie de tensiones entre objeto, modo de corrección y finalidad, revelando que el modo en que se 
corrige resulta ambiguo o no parece favorecer un diálogo eficaz con el destinatario -alumno en tanto no informa unívocamente sobre las condiciones del texto producido. ¿Cómo podría la corrección, así, constituirse en un instrumento necesario para interactuar con los alumnos en su proceso de aprendizaje? La búsqueda personal de cada docente por resolver su tarea de corregir resultaría insuficiente en función de mediar en la enseñanza en el lenguaje y con el lenguaje.

\section{Referencias bibliográficas}

Bota, C. y Bronckart, J.-P. (2010). Voloshinov y Bajtin: dos enfoques radicalmente opuestos de los géneros de textos y de su carácter. En D. Riestra (Comp.), Saussure, Voloshinov y Bajtin revisitados. (pp. 107-127). Buenos Aires: Miño y Dávila

Bronckart, J.-P. (2004). Actividad verbal, textos y discursos. Por un interaccionismo sociodiscursivo. Madrid: Infancia y aprendizaje.

Bronckart, J.-P. (2007). Desarrollo del lenguaje y didáctica de las lenguas. Buenos Aires: Miño y Dávila.

Coromines, J. (2009). Breve diccionario etimológico de la lengua castellana. Buenos Aires: Del Nuevo Extremo y Gredos.

Dolz, J. y Erard, S. (2000). Las actividades metaverbales en la enseñanza de los géneros escritos y orales. En M. Milian y A. Camps, El papel de la actividad metalingüística en el aprendizaje de la escritura (pp. 7-38). Rosario: Homo Sapiens.

Leontiev, A. (1983). El desarrollo del psiquismo. Madrid: Akal.

Real Academia Española (2001). Diccionario de la lengua española. (22 ed). México: Espasa Calpe.

Riestra, D. (2004). Las consignas de trabajo en el espacio socio-discursivo de la enseñanza de la lengua. Tesis de Doctorado. FPE 328. Ginebra: Universidad de Ginebra.

Ruiz, E. M. (1998). Como se corrige redação na escola. Tesis de Doctorado. Universidad de Campiñas, San Pablo.

Vigotsky, L. S. (1930 y 1931/2008). El método instrumental en psicología y Las bases epistemológicas de la psicología. En B. Schneuwly y J-P Bronckart (Coords.), Vigotsky hoy (pp. 21-53). Madrid: Ministerio de Educación y Editorial Popular.

Vigotski, L. S. (1934/2007). Pensamiento y habla. Buenos Aires: Colihue.

Voloshinov, V. N. (1929/2009). El marxismo y la filosofía del lenguaje. Buenos Aires: Godot.

Información de la autora: Stella Maris Tapia es profesora y licenciada en Letras (Universidad de Buenos Aires, Argentina). Es doctora en Ciencias de la Educación (Universidad Nacional de Córdoba, tesis defendida en diciembre de 2014) y profesora adjunta en las áreas de Formación docente y Lectura y Escritura académica, Universidad Nacional de Río Negro (Sede Andina).

E-mail: stapia@unrn.edu.ar

\footnotetext{
${ }^{1}$ El apellido del autor oscila, en las traducciones al castellano, entre las formas Vigotsky, Vigostki y Vigotski. Se ha optado por preservar cómo aparece en las referencias bibliográficas correspondientes, aunque ello atenta contra la unificación de la forma del apellido a lo largo del artículo.

${ }^{2}$ Con autorización de los docentes y autoridades para publicar el nombre de los centros.

${ }^{3}$ La transcripción corresponde a la forma del español voseante propia de la variedad rioplatense.

Para citar este artículo:

Tapia, S.M. (2016). Correcciones en lengua: modos de intervención docente en los textos escritos por los alumnos. Bellaterra Journal of Teaching \& Learning Language \& Literature, 9(3): 69-84.

DOI: http://dx.doi.org/10.5565/rev/jlt3.640
} 